\title{
Evidenz für eine frühzeitige Therapie der COPD mit Tiotropium
}

\author{
Early Treatment of COPD with Tiotropium
}

Autoren

Institute
R. Buhl ${ }^{1}$, T. Welte ${ }^{2}$, C. Vogelmeier ${ }^{3}$, A. Gillissen ${ }^{4}$, T. Voshaar ${ }^{5}$, H. Kögler ${ }^{6}$, D. Liu ${ }^{6}$, T. Glaab ${ }^{1,6}$

Die Institutsangaben sind am Ende des Beitrags gelistet. eingereicht $\quad$ 8.2. 2012 akzeptiert nach Revision 7. 8.2012

\section{Bibliografie}

Dol http://dx.doi.org/ 10.1055/s-0032-1310281 Online-Publikation: 25.9.2012 Pneumologie 2012; 66: 589-595 (c) Georg Thieme Verlag KG Stuttgart $\cdot$ New York ISSN 0934-8387

\section{Korrespondenzadresse}

Prof. Dr. Roland Buhl

Universitätsmedizin der Johannes-Gutenberg Universität Mainz

III. Med. Klinik, Schwerpunkt Pneumologie Langenbeckstraße 1 55131 Mainz r.buhl@3-med.klinik.uni-mainz.de

\section{Zusammenfassung \\ $\nabla$}

Hintergrund: Die Wirksamkeit von Tiotropium versus Kontrolle wurde in einer präspezifizierten Subgruppenanalyse der 4-Jahres-Studie „Understanding Potential Long-term Impacts on Function with Tiotropium“ bei Patienten mit chronisch obstruktiver Lungenerkrankung (COPD) mittleren Schweregrades (GOLD II) untersucht und erstmals den gepoolten Ergebnissen aus den schwereren Krankheitsstadien (GOLD III/IV) gegenübergestellt.

Methode: Randomisierte multizentrische, doppelblinde, kontrollierte Studie mit parallelem Gruppenvergleich bei 5993 Patienten über 4 Jahre. Die Patienten erhielten entweder Tiotropium $18 \mu \mathrm{g}$ einmal täglich oder Plazebo. Als Endpunkte wurden der jährliche $\mathrm{FEV}_{1}$-Abfall und weitere Lungenfunktionsparameter, der Gesundheitsstatus, Exazerbationen sowie die Gesamtletalität erfasst.

Ergebnisse: 46\% der Patientenpopulation befanden sich bei Studienbeginn im mittelgradigen COPD-Stadium GOLD II (Tiotropium: $n=1384$, Kontrolle: $n=1355)$ mit einer mittleren postbronchodilatatorisch gemessenen $\mathrm{FEV}_{1}$ von 1,63 $(0,37)$ L (59\% des Solls). Bei diesen Patienten verbesserte Tiotropium im Vergleich zur Kontrolle die absoluten $\mathrm{FEV}_{1}$-Werte an jedem Studienzeitpunkt vor Bronchodilatation um 101-119ml und nach Bronchodilatation um 52-82 ml (jeweils $\mathrm{p}<0,001$ ). Auch der jährliche (postbronchodilatatorische) $\mathrm{FEV}_{1}$-Abfall war unter Tiotropium signifikant geringer (43 (2) vs. 49 (2) ml/Jahr; p= $0,024)$. Darüber hinaus wurden in der Tiotropiumgruppe die jährliche Exazerbationsrate (Tiotropium: 0,56, Kontrolle: 0,7; p<0,0001), die Zeit bis zur ersten Exazerbation (Tiotropium: 23,09, Kontrolle: 17,47 Monate; p<0,0001) sowie der Gesundheitsstatus (Tiotropium vs. Kontrolle: SGRQ-Verbesserung 2,7 - 4 Einheiten; $\mathrm{p}<0,0001$ ) statistisch signifikant verbessert.

\section{Abstract \\ $\nabla$}

Background: In a prespecified subgroup analysis of the 4-year trial "Understanding Potential Long-term Impacts on Function with Tiotropium", the efficacy of tiotropium versus control in patients with moderate COPD (GOLD II) was examined and compared with the pooled results of patients with more severe disease (GOLD III/IV). Methods: Randomised, multicentre, doubleblind, placebo-controlled, parallel-group study in 5993 patients over a period of 4 years. Patients received either tiotropium $18 \mu \mathrm{g}$ or placebo oncedaily. The study endpoints were the annual $\mathrm{FEV}_{1}$ decline as well as lung function parameters, health status, exacerbations and all-cause mortality.

Results: $46 \%$ of the patients had moderate disease (GOLD II; tiotropium: $n=1384$, control group: $n=$ 1355 ) with a mean postbronchodilator $\mathrm{FEV}_{1}$ of 1.63 (0.37) L (59\% predicted). In these patients with moderate COPD, tiotropium significantly improved the absolute $\mathrm{FEV}_{1}$ values (pre-bronchodilator $\mathrm{FEV}_{1}$ : $101-119 \mathrm{ml}$, post-bronchodilator $\mathrm{FEV}_{1}: 52-82 \mathrm{ml}, \mathrm{p}<0.001$ ) and the postbronchodilator $\mathrm{FEV}_{1}$ decline compared with the control patients (43 (2) vs. 49 (2) ml/year; p=0.024). In addition, there was a statistically significant improvement in the annual exacerbation rate (tiotropium: 0.56 , control: $0.7 ; \mathrm{p}<0.0001$ ), the time to first exacerbation (tiotropium: 23.09, control: 17.47 months; $\mathrm{p}<0.0001$ ) and health status (tiotropium vs. control: minus $2.7-4$ units; $\mathrm{p}<0.0001$ ) in the tiotropium group.

Conclusions: The results of this subgroup analysis support current guideline recommendations and indicate that already patients with moderate COPD (GOLD stage II) benefit clinically from treatment with tiotropium. 
Schlussfolgerungen: Die Ergebnisse dieser Subgruppenanalyse weisen darauf hin, dass bereits Patienten mit mittelgradiger COPD (GOLD-Stadium II) von einer Therapie mit Tiotropium klinisch profitieren, und unterstützen somit aktuelle Leitlinienempfehlungen für eine Dauertherapie mit einem langwirksamen Anticholinergikum.

\section{Einleitung}

\section{$\nabla$}

Die aktuelle nationale COPD-Leitlinie empfiehlt langwirksame Anticholinergika und/oder langwirksame 32 -Sympathomimetika als Mittel der ersten Wahl zur medikamentösen Dauertherapie der COPD ab dem Stadium II (mittelschwere COPD) nach der Klassifikation der „Global Initiative for Obstructive Lung Disease“ (GOLD) [1 - 2]. Die meisten der Studien, die diesen Empfehlungen zugrunde liegen, wurden jedoch insbesondere bei Patienten mit schwerer bis sehr schwerer COPD durchgeführt (GOLD-Stadien III und IV). Damit ist die Evidenzbasis zur bronchodilatatorischen Therapie für die leichteren COPD-Stadien, die auch in der Praxis zahlenmäßig eine viel größere Rolle spielen, noch lückenhaft. Eine Reihe kleinerer Kurzzeitstudien deuten darauf hin, dass COPD-Patienten bereits in frühen Krankheitsstadien unter Symptomen ihrer Erkrankung (Husten, Auswurf, Exazerbationen und verringerte körperliche Belastbarkeit) leiden [4-6]. Dennoch ist bislang noch unklar, welcher klinische Stellenwert langwirksamen Bronchodilatatoren in der Dauertherapie bei Patienten mit mittelgradiger COPD zukommt und welche klinischen und funktionellen Parameter sie beeinflussen.

Ziel der vorliegenden präspezifizierten Subgruppenanalyse, basierend auf der 4-Jahres-Langzeitstudie UPLIFT [7], war es daher, erstmals die Wirksamkeit einer Langzeittherapie mit Tiotropium versus Kontrollgruppe, gezielt bei Patienten mit mittelgradiger COPD (GOLD II), im deskriptiven Vergleich der Wirksamkeit bei Patienten mit schwerer bis sehr schwerer COPD (GOLD III+ IV gepoolt) gegenüberzustellen.

\section{Material und Methoden \\ $\nabla$}

Bei der UPLIFT-Studie handelt es sich um eine randomisierte multizentrische, doppelblinde, kontrollierte Studie mit parallelem Gruppenvergleich. An dieser Langzeitstudie über 4 Jahre nahmen 5993 COPD-Patienten aus 37 Ländern und 487 Studienzentren teil. Patienten inhalierten entweder Tiotropium $(18 \mu \mathrm{g})$ oder Plazebo jeweils einmal täglich morgens. Sowohl in der Tiotropiumgruppe $(n=2987)$ als auch der Kontollgruppe $(n=3006)$ waren als Begleitmedikation sämtliche COPD-Medikamente außer inhalativen Anticholinergika erlaubt.

Die Einschlusskriterien der Studie waren: COPD-Diagnose, Alter $\geq 40$ Jahre, Raucheranamnese $\geq 10$ Packungsjahre, FEV $/$ FVC $\leq 70 \%$ und eine postbronchodilatatorische $\mathrm{FEV}_{1} \leq 70 \%$ des Sollwerts. Patienten mit Asthma, Sauerstoff-Langzeittherapie oder COPD-Exazerbationen im Vormonat wurden von der Studie ausgeschlossen. Nach Randomisierung stellten sich die Patienten nach 1 und 3 Monaten und anschließend alle 3 Monate bis zum Ende der 4-jährigen Studienphase in ihrem Studienzentrum vor. Als coprimäre Endpunkte wurden der jährliche präbronchodilatorische und postbronchodilatorische $\mathrm{FEV}_{1}-\mathrm{Abfall}_{\text {gemessen. Als }}$ sekundäre Endpunkte wurden weitere Lungenfunktionsparameter, der Gesundheitsstatus mittels St. George's Respiratory Questionnaire (SGRQ), mittelschwere und schwere Exazerbationen sowie die Gesamtmortalität erfasst.
Lungenfunktion, Exazerbationen und Gesundheitsstatus Die spirometrische Messung der Lungenfunktion wurde gemäß ATS (American Thoracic Society)-Leitlinien zum Zeitpunkt der Randomisierung, nach 1 Monat und dann halbjährlich bis zum Studienende durchgeführt. Im Anschluss an die präbronchodilatatorische Messung der Lungenfunktion wurde die Studienmedikation verblindet verabreicht. Direkt danach erhielt der Patient $80 \mu \mathrm{g}$ Ipratropium und 60 min später $400 \mu \mathrm{g}$ Salbutamol, jeweils mittels HFA-Dosieraerosol. Die postbronchodilatatorische Lungenfunktionsmessung wurde $30 \mathrm{~min}$ nach der Salbutamolgabe durchgeführt.

Der Gesundheitsstatus wurde vor der Lungenfunktionsmessung mit dem SGRQ bestimmt. Das Auftreten von Exazerbationen wurde bei jeder Visite dokumentiert. Eine Exazerbation war definiert als eine akute Verschlechterung bzw. als das Neuauftreten von mehr als einem Symptom (Husten, Sputum, Sputumpurulenz, Giemen oder Dyspnoe) über mindestens 3 Tage, die eine Behandlung mit einem Antibiotikum und/oder einem systemischen Steroid erforderlich machte. Krankenhauspflichtige, d.h. schwere Exazerbationen wurden ebenfalls erfasst.

\section{Mortalität}

Für alle Studienteilnehmer - auch jene, die vor Ablauf der 4-jährigen Studiendauer die Studie abbrachen - wurde der Vitalstatus bis zum Ende der Studiendauer verfolgt. Die Mortalitätsanalyse erfolgte sowohl für die Mortalität während der Behandlungszeit als auch als präspezifizierte intention-to-treat (ITT)-Analyse am Tag 1470 (Nachverfolgung bis Studienende+30 Tage Auswaschperiode).

\section{Statistik}

Die Berechnung des Stichprobenumfangs basierte auf folgenden Annahmen: Differenz im jährlichen $\mathrm{FEV}_{1}$-Abfall von $15 \mathrm{ml}$ zwischen beiden Behandlungsgruppen, eine Standardabweichung von $90 \mathrm{ml} / \mathrm{Jahr}$, eine Abbruchrate von 35\% und eine statistische Power von 90\% mit einem Signifikanzniveau von 5\%. Im Unterschied zu einer retrospektiven Analyse war die Untersuchung der Subgruppe der GOLD-Stadium II-Patienten präspezifiziert, d.h. bereits im Prüfplan und bei der Randomisierung berücksichtigt. Alle randomisierten Patienten, die Studienmedikation erhielten und an mindestens 3 Lungenfunktionsmessungen bzw. mindestens an 2 SGRQ-Messungen nach Randomisierung beteiligt waren, wurden in die Analyse eingeschlossen.

Das Cox'sche Regressionsmodell wurde zur Berechnung von Hazard Ratios verwendet. Die Anzahl an Exazerbationen und die Anzahl an Tagen, an denen ein Ereignis stattfand, wurde zwischen den Studiengruppen mithilfe von relativen Risiken dargestellt. Zugrunde lag das Poisson'sche Regressionsmodell mit Korrektur für Überstreuung. Alle berechneten P-Werte basierten auf zweiseitigen Tests. Es wurde keine Korrektur für multiples Testen vorgenommen. Alle Analysen wurden mit SAS 8.2 berechnet. Eine detaillierte Beschreibung der zugrunde liegenden Methodik und statistischen Analyse liegt in publizierter Form vor [7,8].

\section{Ergebnisse \\ $\nabla$}

\section{Studienpopulation}

$46 \%$ der an UPLIFT teilnehmenden Patienten befanden sich bei Studienbeginn im GOLD-Stadium II, während 53\% den GOLDStadien III oder IV zuzuordnen waren. Die Verteilung der Patienten auf die Subgruppen und Behandlungsarme sowie die Anzahl 


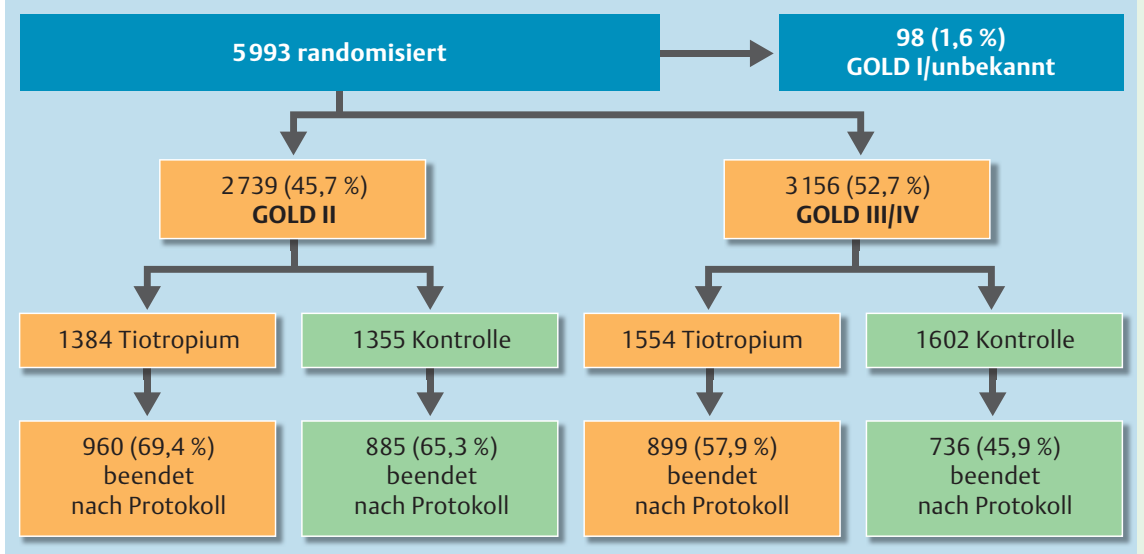

Abb.1 Flussdiagramm mit Verteilung der Patienten auf die Subgruppen und Behandlungsarme. In der GOLD III/IV-Subgruppe brachen mehr Patienten als in GOLD II die Medikamenteneinnahme vor Ablauf der vorgesehenen Behandlungszeit ab, und die Abbruchrate war im Kontroll-Arm um 12 Prozentpunkte höher als im Tiotropium-Arm. Im Schweregrad II hingegen war die Abbruchrate zwischen den beiden Behandlungsgruppen vergleichbar (Differenz: 4,1 Prozentpunkte).

Tab. 1 Baselineparameter.

\begin{tabular}{|c|c|c|c|c|c|}
\hline \multirow{2}{*}{$\begin{array}{l}\text { Parameter } \\
\text { Gruppe }\end{array}$} & & \multicolumn{2}{|c|}{ GOLD II ( $n=2739$ [46\%]) } & \multicolumn{2}{|c|}{ GOLD III/IV (n= 3156 [53\%]) } \\
\hline & & $\begin{array}{l}\text { Tiotropium } \\
(n=1384)\end{array}$ & $\begin{array}{l}\text { Kontrolle } \\
(n=1355)\end{array}$ & $\begin{array}{l}\text { Tiotropium } \\
(n=1554)\end{array}$ & $\begin{array}{l}\text { Kontrolle } \\
(n=1602)\end{array}$ \\
\hline \multicolumn{2}{|l|}{ männlich (\%) } & 72,0 & 72,3 & 78,2 & 75,2 \\
\hline \multicolumn{2}{|l|}{ Alter (J) } & $65(9)$ & $64(9)$ & $65(8)$ & $65(8)$ \\
\hline \multicolumn{2}{|l|}{ Body Mass Index } & $27(5)$ & $27(5)$ & $25(5)$ & $25(5)$ \\
\hline \multirow[t]{2}{*}{ Raucherstatus } & Akt. Raucher (\%) & 30,7 & 35,0 & 28,2 & 25,6 \\
\hline & $\begin{array}{l}\text { Raucheranamnese } \\
\text { (Packungsjahre) }\end{array}$ & $48(28)$ & $47(27)$ & $50(28)$ & $49(29)$ \\
\hline \multicolumn{2}{|l|}{ Dauer der COPD (J) } & $10(8)$ & $9(7)$ & $10(8)$ & $10(7)$ \\
\hline \multicolumn{2}{|c|}{ SGRQ-Gesamtscore (Einheiten) } & $41(17)$ & $42(17)$ & $50(16)$ & $49(17)$ \\
\hline \multirow[t]{2}{*}{$\mathrm{FEV}_{1}(\mathrm{~L})$} & Prä-bd & $1,36(0,37)$ & $1,36(0,35)$ & $0,87(0,27)$ & $0,86(0,28)$ \\
\hline & Post-bd & $1,63(0,37)$ & $1,64(0,36)$ & $1,06(0,29)$ & $1,04(0,30)$ \\
\hline \multirow[t]{2}{*}{$\mathrm{FEV}_{1}(\%$ Soll) } & Prä-bd & $49(8)$ & $49(8)$ & $31(8)$ & $31(8)$ \\
\hline & Post-bd & $59(6)$ & $59(6)$ & $38(8)$ & $38(8)$ \\
\hline \multirow[t]{2}{*}{ Vormedikation (\%) } & LABA* & 55,7 & 55,4 & 63,8 & 64,1 \\
\hline & $\mathrm{ICS}^{*}$ & 58,5 & 56,9 & 64,0 & 65,9 \\
\hline
\end{tabular}

GOLD: Global initiative for Obstructive Lung Disease; LABA: Inhalierbarer langwirksamer $\beta_{2}$-adrenerger Agonist; ICS: Inhalierbares Kortikosteroid. Die Standardabweichung ist in Klammern angegeben. ${ }^{*}$ Kombinationspräparate sind doppelt gelistet (einmal bei jedem Inhaltsstoff).

der Patienten, die die Studie protokollgemäß beendete, ist in - Abb. 1 dargestellt. Tab. 1 zeigt die demografischen Daten sowie die Ausgangsdaten für Gesundheitsstatus, Lungenfunktion und Vormedikation für die Patienten der Subgruppen GOLD II bzw. III/IV. In beiden Subgruppen bestanden keine relevanten Unterschiede zwischen Patienten im Tiotropium- und im Kontroll-Arm.

GOLD II-Patienten wiesen erwartungsgemäß einen höheren Anteil aktiver Raucher auf als GOLD III/IV-Patienten. Auch der SGRQ-Gesamtpunktwert war bei GOLD III/IV-Patienten höher, d. h. schlechter als im Stadium GOLD II. Die Lungenfunktionswerte bei Randomisierung spiegelten die auf dem $\mathrm{FEV}_{1}$-Wert basierende Schweregradeinteilung nach GOLD wider. Über alle GOLD-Schweregrade und in beiden Behandlungsgruppen zeigte sich eine deutliche Teilreversibilität der Atemwegsobstruktion, jeweils gemessen an der $\mathrm{FEV}_{1}$ nach maximaler Bronchodilatation durch $80 \mu \mathrm{g}$ Ipratropiumbromid und $400 \mu \mathrm{g}$ Salbutamol. In beiden Subgruppen bestanden keine wesentlichen Unterschiede zwischen den beiden Behandlungsgruppen hinsichtlich der Vormedikation.

\section{Lungenfunktion}

- Abb. 2 zeigt den $\mathrm{FEV}_{1}$-Verlauf über vier Jahre in den Subpopulationen GOLD II und GOLD III/IV. In der GOLD II-Subpopulation kam es im Vergleich mit der Kontrollgruppe unter Therapie mit
Tiotropium zu einer geringen, statistisch signifikanten Reduktion des postbronchodilatatorischen $\mathrm{FEV}_{1}$-Abfalls (43 (2) $\mathrm{ml} / \mathrm{Jahr}$ gegenüber 49 (2) ml/Jahr; p=0,024). Der jährliche Abfall der präbronchodilatatorisch gemessenen $\mathrm{FEV}_{1}$ sowie der FVC- bzw. VC-Werte unterschied sich zwischen den Gruppen nicht signifikant. Die absoluten prä- und postbronchodilatatorisch gemessenen $\mathrm{FEV}_{1}$-Verbesserungen zwischen 101 und $119 \mathrm{ml}$ bzw. 52 und $82 \mathrm{ml}$ ( $\mathrm{p}<0,001$ an jedem Messzeitpunkt) in der Tiotropiumgruppe gegenüber der Kontrolle bei GOLD II-Patienten wurden über

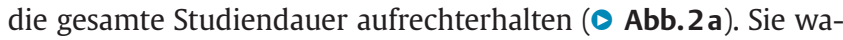
ren höher als in der Subpopulation der GOLD III/IV-Patienten, bei denen, ausgehend von schweregradbedingt niedrigeren Ausgangswerten, Verbesserungen zwischen 71 und $91 \mathrm{ml}$ (präbronchodilatatorisch) bzw. 33 und $57 \mathrm{ml}$ (postbronchodilatatorisch) registriert wurden $(\mathrm{p}<0,001$ an jedem Messzeitpunkt zwischen Tiotropium und Kontrolle) ( $\bullet$ Abb.2b). Der jährliche Abfall der prä- und postbronchodilatatorischen $\mathrm{FEV}_{1}$ sowie der FVC- bzW VC-Werte unterschied sich zwischen den Behandlungsgruppen in den beiden Subgruppen (GOLD II und GOLD III/IV) nicht signifikant.

\section{Gesundheitsstatus}

Tiotropium verbesserte in der GOLD II-Subpopulation über alle Messzeitpunkte (6 Monate nach Randomisierung bis Studienende) den SGRQ-Gesamtpunktwert im Vergleich zur Kontroll- 

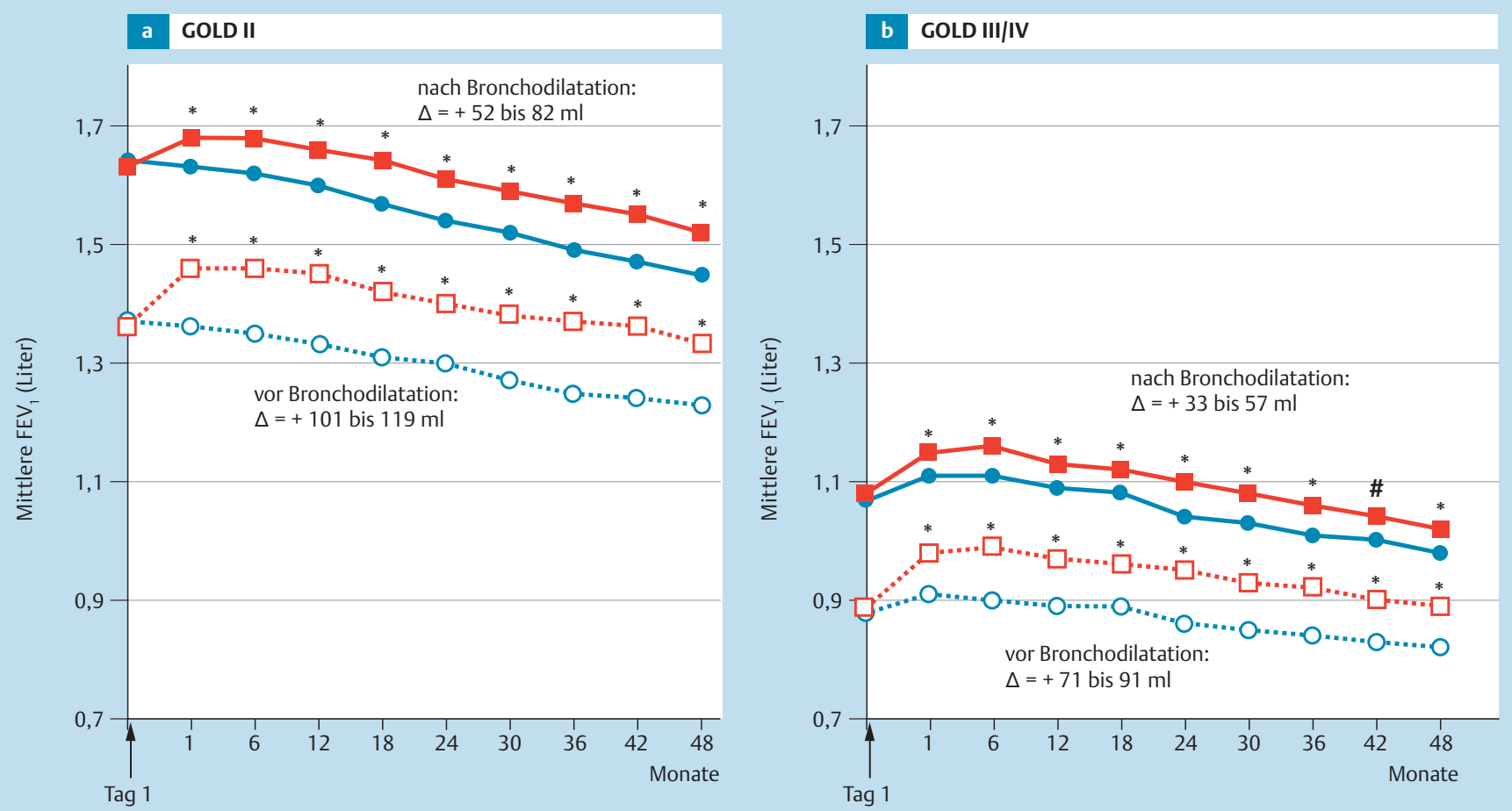

Abb.2 Zeitlicher Verlauf von FEV 1 vor und nach Bronchodilatation in der Tiotropium- und Kontrollgruppe. a Subgruppe der GOLD II-Patienten. b Subgruppe der GOLD III/IV-Patienten. Offene Symbole und punktierte Linien: FEV 1 vor Bronchodilatation; gefüllte Symbole und durchgezogene Linien: FEV 1 nach Bronchodilatation. Blaue Kreise: Kontrollgruppe; rote Quadrate: Tiotropiumgruppe. ${ }^{*} p<0,0001 ;{ }^{*} p=0,0002$. Tiotropium bewirkte in beiden Subgruppen sowohl vor als auch nach Bronchodilatation eine signifikante Zunahme der FEV ${ }_{1}$, die über den Beobachtungszeitraum von vier Jahren erhalten blieb. Die Zunahme in der Tiotropiumgruppe war in der GOLD II-Subgruppe größer als in der GOLD III/IV-Subgruppe.

gruppe signifikant um 2,7-4,0 Einheiten (adjustiert zum Ausgangswert, $\mathrm{p}<0,0001 \mathrm{zu}$ allen Messzeitpunkten). Damit war der SGRQ-Gesamtpunktwert nach 4 Jahren Studiendauer besser als zu Studienbeginn ( $\bullet$ Abb.3a). Die Verbesserung des SGRQ-Gesamtpunktwerts in der Tiotropiumgruppe war in der GOLD IISubpopulation numerisch stärker ausgeprägt als bei den Patienten in der GOLD III/IV-Subpopulation ( $\bullet$ Abb.3 b: SGRQ-Gesamtpunktwert Tiotropium gegenüber Kontrolle: 1,9-2,9 Einheiten; $\mathrm{p}<0,01$ ), die entsprechend ihrem höheren COPD-Schweregrad mit einem schlechteren Ausgangswert in die Studie eintraten. Der SGRQ-Gesamtpunktwert wurde auch in der GOLD III/IV-Subpopulation über 4 Jahre aufrechterhalten $(\boldsymbol{A} \mathbf{A b b} . \mathbf{3 b})$. Wie - Abb. 4 zeigt, war der Anteil der Patienten, die eine Verbesserung des SGRQ-Gesamtpunktwerts um mindestens 4 Punkte erreichten, in beiden Subpopulationen unter Behandlung mit Tiotropium signifikant höher als in den jeweiligen Kontrollgruppen (GOLD II: $p<0,0001$, GOLD III/IV: $p \leq 0,0012$, jeweils an allen Messzeitpunkten).

\section{Exazerbationen}

In der GOLD II- bzw. GOLD III/IV-Subpopulation entwickelten 65\% bzw. 70\% der Patienten im Studienverlauf mindestens eine Exazerbation. In beiden Subgruppen (GOLD II und GOLD III/IV) reduzierte Tiotropium, verglichen mit der Kontrollbehandlung, signifikant die Exazerbationsrate ( $\bullet$ Abb.5). Bei GOLD II-Patienten nahm die jährliche Exazerbationsrate unter Tiotropium um 20\% ab (Tiotropium: 0,56, Kontrolle: 0,70 Exazerbationen pro Patient und Jahr; $\mathrm{p}<0,0001$ ), während die relative Ratenreduktion durch Tiotropium bei GOLD III/IV-Patienten $12 \%$ betrug (jährliche Exazerbationsrate Tiotropium: 0,88, Kontrolle: 0,99; $p=0,0019$ ). Bei der Berechung der number-needed-to-treat (NNT) zeigte sich, dass 7,1 Patienten im GOLD-Stadium II mit Tiotropium behandelt werden mussten, um eine Exazerbation pro Jahr zu verhindern, während die NNT in den Stadien III/IV 9,1 Patienten betrug. In beiden Subgruppen (GOLD II; GOLD III/IV) waren die Rate und der Anteil von Patienten mit krankenhauspflichtigen Exazerbationen gegenüber den Kontrollgruppen nicht signifikant unterschiedlich. Die Zeit bis zur ersten Exazerbation wurde in der Tiotropiumgruppe gegenüber der Kontrollgruppe in der GOLD II-Subpopulation signifikant um 5,62 Monate (Tiotropium: Median 23,09 Monate, Kontrolle: Median 17,47 Monate; p<0,0001) und in der GOLD III/IV-Subpopulation um 2,3 Monate (Tiotropium: Median 11,99 Monate, Kontrolle: Median 9,69 Monate; $\mathrm{p}=0,0038$ ) verlängert.

\section{Gesamtletalität}

Die Hazard Ratio [HR, 95\%-Konfidenzintervall (95\% CI)] für Gesamtletalität in der Tiotropium- versus der Kontrollgruppe betrug bei den COPD-Patienten im Schweregrad GOLD II während der Behandlungszeit 0,85 (95\% CI 0,66; 1,09) und in der ITT-Analyse 0,88 (95\% CI 0,69; 1,11). Für die COPD-Patienten im Schweregrad III/IV betrug die HR während der Behandlungszeit 0,84 $(95 \% \mathrm{Cl} 0,71 ; 1,0)$ und in der präspezifizierten ITT-Analyse 0,93 (95\% CI 0,79; 1,08). Die schweren unerwünschten Ereignisse wurden bereits an anderer Stelle publiziert [7].

\section{Diskussion \\ $\nabla$}

Im Unterschied zu einer bereits publizierten Subgruppenanalyse der UPLIFT-Studie [9] konzentriert sich die vorliegende Analyse nicht auf die einzelnen Stadien der COPD nach GOLD, sondern auf den deskriptiven Vergleich der Wirksamkeit und Verträglichkeit von Tiotropium versus Kontrolle zwischen mittelgradiger 

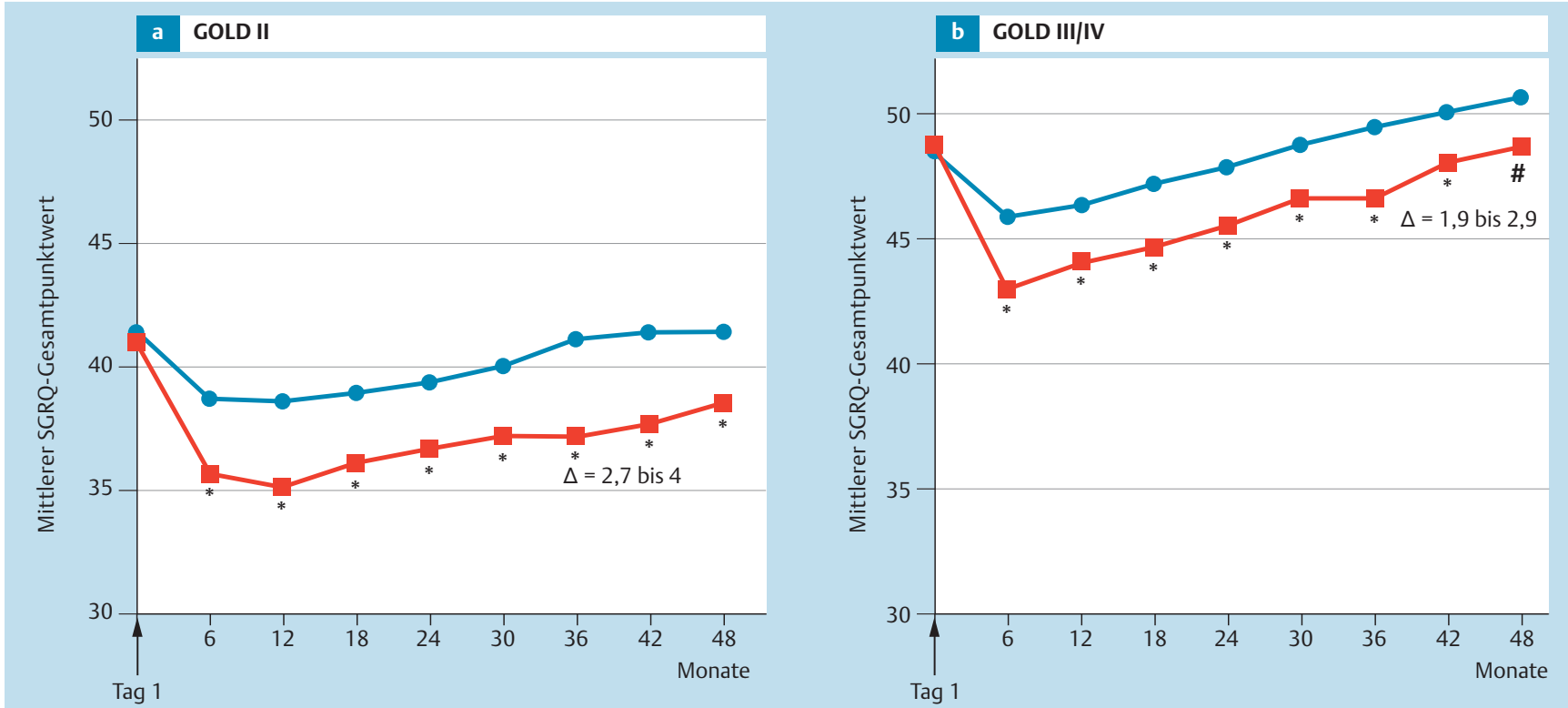

Abb.3 Verlauf des Gesundheitsstatus (SGRQ-Gesamtpunktwert) über vier Jahre. a Subgruppe der GOLD II-Patienten. b Subgruppe der GOLD III/IV-Patienten. Blaue Kreise: Kontrollgruppe; rote Quadrate: Tiotropiumgruppe. ${ }^{*} p<0,001 ;{ }^{\S} p=0,004 ;{ }^{\sharp} p=0,006$. In beiden Subgruppen bewirkte Tiotropium eine Reduktion des SGRQ-Gesamtpunktwerts (Verbesserung des Gesundheitsstatus), die über die gesamte Studiendauer von vier Jahren erhalten blieb. Die Differenz durch Tiotropium betrug in der GOLD III/IV-Subgruppe zwischen 1,9 und 2,9 Einheiten, während sie in der GOLD II-Subgruppe mit Werten zwischen 2,7 und 4,0 größer war.

COPD (GOLD II) und schwerer/sehr schwerer COPD (gepoolte GOLD III+IV-Daten). Diese präspezifizierte Subgruppenanalyse aus UPLIFT ist besonders deshalb interessant, da sie an einer großen Population von Patienten mit mittelschwerer COPD (GOLD II) über 4 Jahre durchgeführt wurde und den Einsatz von langwirksamen Anticholinergika bei COPD-Patienten im Stadium GOLD II auf eine breitere Evidenzbasis stellt. GOLD II-Patienten stellen auch in Deutschland die mit Abstand größte Subpopulation von COPD-Patienten dar [10]. Bereits in diesem relativ frühen Stadium der Erkrankung leiden die Patienten unter Exazerbationen und einem reduzierten Gesundheitsstatus, wie auch die Ergebnisse dieser Untersuchung zeigen [6,11].

Die absoluten Verbesserungen von $\mathrm{FEV}_{1}$ und Gesundheitsstatus sowie die Prävention von Exazerbationen waren unter Tiotropium bei Patienten mit mittelgradiger COPD (GOLD II) ausgeprägter als bei Patienten mit schwerer bzw. sehr schwerer COPD (GOLD III/IV). Diese Ergebnisse belegen erstmals an einer großen und gut definierten Population, dass COPD-Patienten bereits in frühen Stadien (ab GOLD-Stadium II) von einer Therapie mit Tiotropium klinisch profitieren, und unterstützen gleichlautende Empfehlungen in aktuellen Leitlinien [1,12]. Es stellt sich in diesem Kontext zunächst die Frage nach der Relevanz einer im Verhältnis höheren postbronchodilatatorischen Verbesserung der pulmonalen Funktion im GOLD-Stadium II gegenüber einer geringeren Verbesserung bei schwerer und sehr schwerer COPD. Aus pathophysiologischer Perspektive lässt sich die Ergebniskonstellation gut mit den Zeitachsen der COPD-Pathogenese vereinbaren. Mit fortschreitenden COPD-typischen Lungenveränderungen kommt es sowohl zu einem zunehmenden Verlust an Lungenfunktion als auch zu einer abnehmenden Reversibilität der obstruktiven Komponente der Ventilationsstörung [13]. Als klinische Konsequenz unterstreichen die Ergebnisse die Forderung nach frühzeitiger und konsequenter bronchodilatatorischer Therapie, um die mit der Limitierung des Atemflusses bei COPD sehr häufig einhergehende Vermeidung körperlicher Aktivität und konsekutive De- konditionierung so weit und so lange als möglich aufzuhalten [4]. Paradigma der Gültigkeit dieses Prinzips sind die additiven Effekte von Rehabilitation und anticholinerger Therapie [14]. In gleicher Weise belegt diese Analyse erneut und über einen Zeitraum von 4 Jahren, dass auch in fortgeschrittenen COPD-Stadien eine klinische Wirkung einer langwirksamen anticholinergen Bronchodilatation zu erwarten ist. Neben Parametern der pulmonalen Funktion wurden durch Tiotropium auch patientenrelevante Endpunkte wie der mittels SGRQ gemessene Gesundheitsstatus über die gesamte Studiendauer von 4 Jahren positiv beeinflusst. Die tatsächliche klinische Bedeutung der im Mittel absolut nur geringen Verbesserungen der SGRQ-Scores erschließt sich im Vergleich zwischen Studienbeginn und -ende und bei Betrachtung des absoluten Anteils der Patienten mit einer Verbesserung des SGRQ-Scores um mindestens 4 Punkte, der Schwelle eines klinisch relevanten Unterschieds [15]. So war der Gesundheitsstatus der mit Tiotropium behandelten Patienten selbst am Ende der 4-jährigen Studienzeit noch besser als zu Studienbeginn. Die absoluten Verbesserungen der SGRQ-Scores und der Anteil an Patienten mit einer Abnahme der Scores um mindestens 4 Punkte waren bei GOLD II-Patienten wiederum ausgeprägter als bei GOLD III/IV-Patienten. Auch der Gesundheitsstatus spiegelt somit sowohl die im Verlauf der COPD progrediente Verschlechterung dieses Parameters als auch das mit Fortschreiten der Erkrankung abnehmende Verbesserungspotential. Die SGRQ-Analyse belegt den überlegenen Effekt einer frühzeitigen bronchodilatatorischen Therapie im Verhältnis zur Bronchodilatation erst bei fortgeschrittener Erkrankung. Wiederum liegt der Schluss nahe und sollte durch klinische Prüfungen bestätigt werden, dass durch konsequente Bronchodilatation bei vielen Patienten die körperliche Belastbarkeit und der Gesundheitszustand länger auf höherem Niveau erhalten werden kann.

Schließlich wirft die Prävention von Exazerbationen durch Therapie mit Tiotropium schon im Stadium II nach GOLD eine ganze Reihe von Fragen auf. Prinzipiell können Exazerbationen in allen 

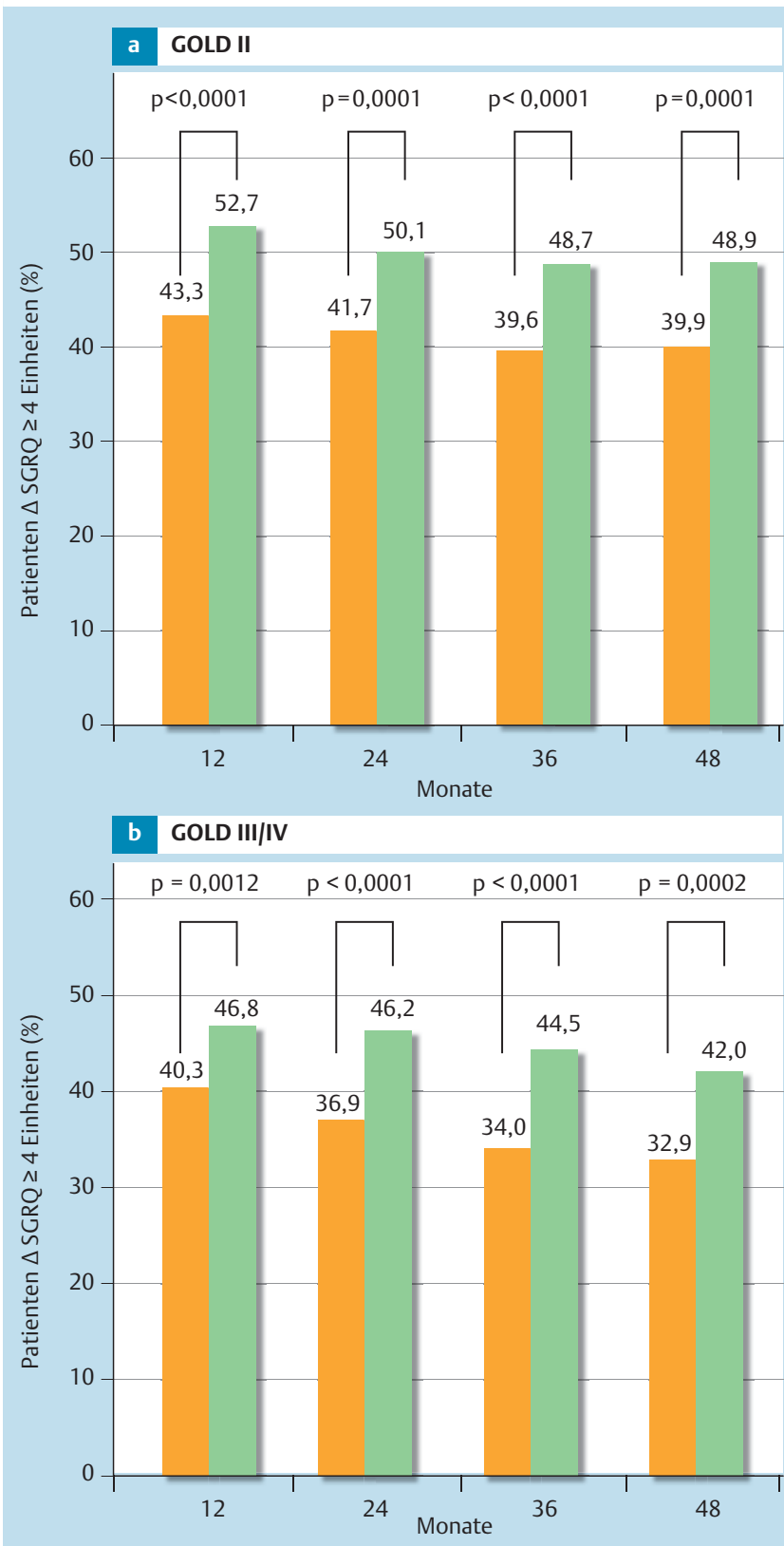

Abb.4 Anteil der Patienten mit Abnahme (d.h Verbesserung) des SGRQGesamtpunktwerts um $\geq 4$ Einheiten gegenüber dem Ausgangswert nach 1, 2, 3 und 4 Jahren. a Subgruppe der GOLD II-Patienten. b Subgruppe der GOLD III/IV-Patienten. Orange Balken: Kontrollgruppe; grüne Balken: Tiotropiumgruppe. Signifikanzniveau wie angegeben. In beiden Subgruppen war der Anteil der Patienten, die gegenüber dem Ausgangswert eine klinisch relevante Abnahme des SGRQ-Gesamtpunktwerts erlebten, zu jedem Zeitpunkt unter Tiotropium signifikant höher als in der Kontrollgruppe.

Krankheitsstadien auftreten, sodass deren Vermeidung in jedem Krankheitsstadium für Arzt und Patient ein sehr wichtiges Therapieziel darstellt $[1,2,11,16]$. Die negativen Konsequenzen von Exazerbationen bei COPD stehen außer Zweifel. Für Patienten mit fortgeschrittener COPD können Exazerbationen eine vitale Bedrohung darstellen [11]. Dies bedeutet anderseits nicht, dass Exazerbationen für Patienten mit leichter ausgeprägter Erkrankung nicht ebenso klinisch relevant sind. Nicht wenige dieser Patienten stehen noch im Berufsleben oder sind anderweitig aktiv, sodass die Prävention von Exazerbationen als in dieser Population ebenso relevant eingeschätzt werden muss. Die letztlich zu Dekondi-

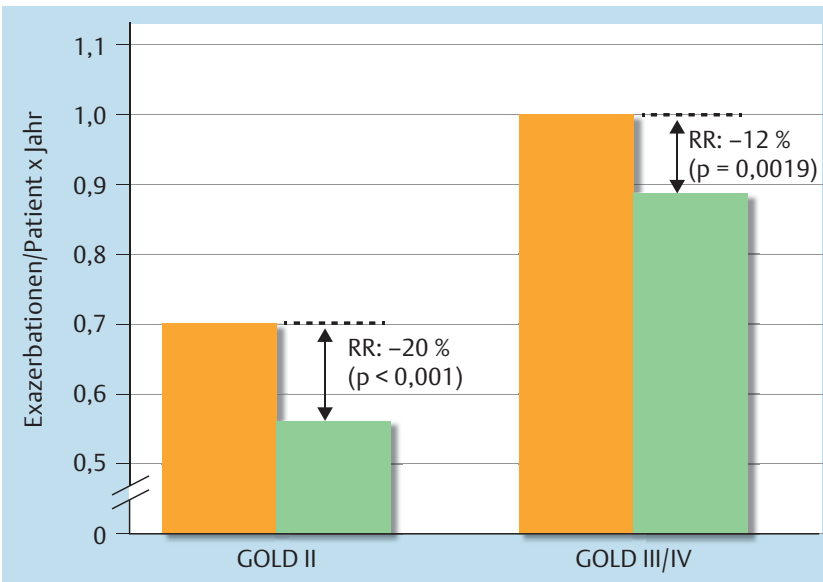

Abb.5 Effekt von Tiotropium auf die jährliche Exazerbationsrate. Links: Subgruppe der GOLD II-Patienten; rechts: Subgruppe der GOLD III/IV-Patienten. Orange Balken: Kontrollgruppe; grüne Balken: Tiotropiumgruppe. Tiotropium bewirkte in beiden Subgruppen eine signifkante Reduktion der Exazerbationsrate (GOLD II: Tiotropium: 0,56, Kontrolle: 0,7 Exazerbationen pro Patient und Jahr; $\mathrm{p}<0,0001$; GOLDIII/IV: Tiotropium: 0,88, Kontrolle: 0,99 Exazerbationen pro Patient und Jahr; $p=0,0019)$. Der Effekt von Tiotropium vs Kontrolle war sowohl relativ (relative Reduktion der Rate um $20 \%$ gegenüber $12 \%$ ) als auch absolut (absolute Reduktion der Rate um 0,14 gegenüber 0,11 Exazerbationen pro Patient und Jahr) ausgeprägter in der Subgruppe der GOLD II-Patienten als bei den GOLD III/IV-Patienten.

tionierung und Invalidität führende Abwärtsspirale sollte so früh als möglich durch effektive bronchodilatative Therapie unterbrochen werden, idealerweise verbunden mit Anstrengungen zur Raucherentwöhnung. Diese Aktualität dieser Argumente wird noch unterstrichen durch die hohe Gewichtung von Exazerbationen als Risikoindikator im neuen GOLD-Strategiepapier [12]. Die Patientenzahl, die mit Tiotropium behandelt werden musste, um eine Exazerbation pro Jahr zu vermeiden (NNT), lag bei UPLIFT in der Subpopulation von Patienten mit mittelgradiger COPD (GOLD II) bei 7,1 und damit niedriger als bei den Patienten mit schwerer COPD. Im Vergleich dazu mussten zum Beispiel 52 Patienten über 6 Jahre mit dem Cholesterinsynthesehemmer Pravastatin behandelt werden, um einen Myokardinfarkt zu vermeiden [17].

Die in der UPLIFT-Studie mit Tiotropium erzielten Behandlungseffekte waren unabhängig von der medikamentösen Begleittherapie. In diesem Zusammenhang ist darauf hinzuweisen, dass sowohl in der Tiotropiumgruppe als auch in der Kontrollgruppe mit Ausnahme von Anticholinergika sämtliche respiratorische Begleitmedikation inklusive langwirksamer $\beta 2$-Sympathomimetika, inhalativer Steroide sowie deren Kombination erlaubt waren [7]. Dies ist ein wichtiger Unterschied zur zweiten großen COPDLangzeitstudie TORCH [18].

Die Therapie-Abbruchrate war in den beiden Behandlungsarmen der GOLD II-Subpopulation vergleichbar, sodass die Interpretation der Ergebnisse nicht durch eine zwischen den Gruppen unterschiedliche Abbruchrate beeinflusst wurde. Die Reversibilität der Atemwegsobstruktion nach Gabe eines Bronchodilatators war über alle Schweregrade signifikant und wies für das GOLDStadium II die höchsten Werte auf. Dieser bereits wiederholt diskutierte Befund belegt, dass die Diagnose einer COPD bzw. eines Asthmas nicht allein auf das Kriterium der Reversibilität gestützt werden kann $[19,20]$. Diese Schlussfolgerung ist im aktuellen Strategiepapier von GOLD erstmals umgesetzt [12] .

Insgesamt bietet die vorliegende präspezifizierte Subgruppenanalyse auf Basis der größten bislang in einer Interventionsstudie 
untersuchten Population von Patienten mit mittelgradiger COPD eine solide Evidenzgrundlage für den leitliniengerechten Einsatz langwirkender Bronchodilatatoren, im vorliegenden Fall von Tiotropium bei COPD-Patienten ab dem Stadium GOLD II. Diese Ergebnisse für GOLD II-Patienten waren in dieser Ausprägung nicht unbedingt zu erwarten, wenn man bedenkt, dass Patienten mit mittelgradiger COPD noch relativ gute Lungenfunktionswerte, einen meist nur mäßig eingeschränkten Gesundheitsstatus und seltener Exazerbationen aufweisen, als dies in den schweren Krankheitsstadien der Fall ist.

Ob langwirksame Anticholinergika den langwirksamen $\beta 2$-Sympathomimetika bei der therapeutischen Wirksamkeit überlegen sind, kann aus der UPLIFT-Studie nicht abgeleitet werden, da keine Randomisierung bezüglich der Begleitmedikation vorgenommen wurde. In der kürzlich publizierten POET-COPD-Studie konnte erstmals im direkten Vergleich gezeigt werden, dass Tiotropium dem langwirksamen $\beta 2$-Sympathomimetikum Salmeterol bei der Reduktion aller untersuchten Exazerbationsendpunkte signifikant überlegen war [21].

Zusammenfassend zeigt die vorliegende UPLIFT-Subgruppenanalyse, dass COPD-Patienten im Schweregrad GOLD II klinisch relevante Symptome und Exazerbationen aufweisen. Dies unterstreicht die Bedeutung einer frühzeitigen Therapie symptomatischer Patienten. Das langwirksame Anticholinergikum Tiotropium verbesserte bei COPD-Patienten auch im GOLD-Stadium II unabhängig von der Begleitmedikation signifikant die Lungenfunktion, den Gesundheitsstatus sowie das Exazerbationsrisiko während der gesamten Studiendauer von 4 Jahren und unterstützt die aktuelle GOLD-Position einer frühzeitigen Therapie von COPD-Patienten mit Tiotropium als Mittel der 1. Wahl ab der Kategorie B [12].

\section{Interessenkonflikt}

$\nabla$

R. Buhl hat Honorare für Vorträge und/oder Beratungen und/oder Erstattungen von Kosten für den Besuch von Kongressen oder Fortbildungsveranstaltungen von AstraZeneca, Boehringer Ingelheim, Chiesi, GlaxoSmithKline, Grifols, Merck Sharpe \& Dohme, Mundipharma, Novartis und Nycomed erhalten.

T. Welte hat Honorare für Vorträge und/oder Beratungen und/ oder Erstattungen von Kosten für den Besuch von Kongressen oder Fortbildungsveranstaltungen von AstraZeneca, Boehringer Ingelheim, GILEAD, GlaxoSmithKline, Grifols, Infectopharm, Merck Sharpe \& Dohme, Novartis, Nycomed und Pfizer erhalten. C. Vogelmeier hat Honorare für Vorträge und/oder Beratungen und/oder Erstattungen von Kosten für den Besuch von Kongressen oder Fortbildungsveranstaltungen von AstraZeneca, Boehringer Ingelheim, Chiesi, GlaxoSmithKline, Grifols, Janssen-Cilag, Mundipharma, Novartis, Nycomed und Pfizer erhalten.

A. Gillissen hat Honorare für Vorträge und/oder Beratungen und/ oder Erstattungen von Kosten für den Besuch von Kongressen oder Fortbildungsveranstaltungen von Boehringer Ingelheim, Chiesi, GlaxoSmithKline, Mundipharma, Novartis und Nycomed erhalten.

T. Voshaar hat Honorare für Vorträge und/oder Beratungen und/ oder Erstattungen von Kosten für den Besuch von Kongressen oder Fortbildungsveranstaltungen von Boehringer Ingelheim, Chiesi, Janssen-Cilag, GlaxoSmithKline, Mundipharma, Novartis und Teva erhalten.

H. Kögler, D. Liu und T. Glaab sind Angestellte von Boehringer Ingelheim.
Institute

${ }^{1}$ Universitätsmedizin der Johannes-Gutenberg Universität, III. Med. Klinik, Schwerpunkt Pneumologie, Mainz

${ }^{2}$ Medizinische Hochschule Hannover, Abteilung Pneumologie, Hannover

${ }^{3}$ Universitätsklinikum Gießen und Marburg, Klinik für Innere Medizin mit Schwerpunkt Pneumologie, Marburg

${ }^{4}$ Klinikum Kassel, Klinik für Lungen und Bronchialmedizin, Kassel

${ }^{5}$ Krankenhaus Bethanien, Med. Klinik III, Lungenzentrum, Moers

${ }^{6}$ Boehringer Ingelheim, Medizinische Wissenschaft und Klinische Biostatistik

\section{Literatur}

1 Vogelmeier C, Buhl R, Criée CP et al. Leitlinie der Deutschen Atemwegsliga und der Deutschen Gesellschaft für Pneumologie und Beatmungsmedizin zur Diagnostik und Therapie von Patienten mit chronisch obstruktiver Bronchitis und Lungenemphysem (COPD). Pneumologie 2007; 61: e1 - e40

2 Rabe KF, Hurd S, Anzueto A et al. Global strategy for the diagnosis, management and prevention of COPD: GOLD executive summary. Am J Respir Crit Care Med 2007; 176: 532 - 555

3 Celli BR, MacNee W. ATS/ERS Task Force. Standards for the diagnosis and treatment of patients with COPD: a summary of the ATS/ERS position paper. Eur Respir J 2004; 23: 932 -946

4 Watz H, Waschki B, Meyer $T$ et al. Physical activity in patients with COPD. Eur Respir J 2009; 33: 262 - 272

5 Ofir D, Laveneziana P, Webb KA et al. Mechanisms of dyspnea during cycle exercise in symptomatic patients with GOLD stage I chronic obstructive pulmonary disease. Am J Respir Crit Care Med 2008; 177: $622-629$

6 van Wetering $C R$, Hoogendoorn M, Mol SM et al. Short- and long-term efficacy of a community-based COPD management programme in less advanced COPD: a randomized controlled trial. Thorax 2010; 65: 7-13

7 Tashkin DP, Celli B, Senn S et al. A 4-year trial of tiotropium in chronic obstructive pulmonary disease. N Engl J Med 2008; 359: 1543 - 1554

8 Decramer M, Celli B, Tashkin DP et al. Clinical trial design considerations in assessing long-term functional impacts of tiotropium in COPD: the UPLIFT trial. COPD 2004; $1: 303-312$

9 Decramer $M$, Celli B, Kesten $S$ et al. Effect of tiotropium on outcomes in patients with moderate chronic obstructive pulmonary disease (UPLIFT): a prespecified subgroup analysis of a randomised controlled trial. Lancet 2009; 374: $1171-1178$

10 Geldmacher H, Biller H, Herbst A et al. COPD-Prävalenz in Deutschland Ergebnisse der BOLD Studie. Dtsch Med Wochenschr 2008; 133: 2609-2614

11 Soler-Cataluña JJ, Martínez-García MA, Román Sánchez P et al. Severe acute exacerbations and mortality in patients with chronic obstructive pulmonary disease. Thorax 2005; 60: 925 -931

12 Global Initiative for Chronic Obstructive Lung Disease. Global Strategy for the diagnosis, management, and prevention of chronic obstructive pulmonary disease. Global Initiative for Chronic Obstructive Lung Disease website: 2011: http://www.goldcopd.org

13 Beaucage F, Frémault $A$, Janssens $W$ et al. FEV $_{1}$ decline in COPD patients according to severity stages. Am J Respir Crit Care Med 2008; 177: A401

14 Casaburi R, Kukafka D, Cooper CB et al. Improvement in exercise tolerance with the combination of tiotropium and pulmonary rehabilitation in patients with COPD. Chest 2005; 127: 809-817

15 Jones $P W$. Interpreting thresholds for a clinically significant change in health status in asthma and COPD. Eur Respir J 2002; 19: 398 - 404

16 Beeh KM, Beier J, Buhl R et al. Wirksamkeit von Tiotropium (Spiriva ${ }^{\circledR}$ ) bei verschiedenen Schweregraden der COPD. Pneumologie 2006; 60: $341-346$

17 The LIPID Study Group. Long-term effectiveness and safety of pravastatin in 9014 patients with coronary heart disease and average cholesterol concentrations: the LIPID trial follow-up. Lancet 2002; 359: 1379 1387

18 Calverley $P$, Anderson JA, Celli B et al. Salmeterol and fluticasone propionate and survival in chronic obstructive pulmonary disease. $\mathrm{N}$ Engl J Med 2007; 356: 775 - 789

19 Calverley PM, Rennard SI. What have we learned from large drug treatment trials in COPD? Lancet 2007; 370: 774-785

20 Tashkin DP, Celli B, Decramer $M$ et al. Bronchodilator responsiveness in patients with COPD. Eur Respir J 2008; 31: 742 - 750

21 Vogelmeier C, Hederer B, Glaab T et al. Tiotropium versus salmeterol for the prevention of exacerbations in COPD. N Engl J Med 2011; 364: $1093-1103$ 